\title{
ALTERAÇÕES DO PROGRAMA DE MATEMÁTICA DO ENSINO BÁSICO PORTUGUÊS: O CASO DO TEMA ORGANIZAÇÃO E TRATAMENTO DE DADOS
}

\author{
Maria Palmira Alves \\ José António Fernandes \\ Universidade do Minho
}

\section{Resumo}

Neste estudo analisam-se as alterações ocorridas no recentemente aprovado Programa de Matemática para o Ensino Básico português(2013), com foco no tema Organização e Tratamento de Dados, relativamente ao programa anterior (2007), tendo por referência as recomendações atuais para o ensino da estatística. Para tal, procedeu-se a uma análise de conteúdo dos dois programas escolares, primeiro em um nível estrutural e, seguidamente, focada no tema Organização e Tratamento de Dados. Em termos de resultados obtidos, diferentemente do programa anterior, no programa de 2013 omitemse indicações metodológicas e recursos, que se traduzem na não referência aos projetos investigativos, ao trabalho de grupo e às calculadoras $\mathrm{e}$ computadores, revelando um retrocesso relativamente às recomendações atuais para a abordagem desta temática.

Palavras-chave: Programas de matemática; ensino básico; organização e tratamento de dados. 


\title{
CHANGES IN THE MATH SYLLABUS OF THE PORTUGUESEBASIC EDUCATION: THE CASE OF THE TOPIC ORGANISATION AND DATA PROCESSING
}

\begin{abstract}
In this studythe changes in the recently approved Math Syllabus for the Portuguese Basic Education (2013) are analysed, focusing on the topic Organisation and Data Processing, related to the previously syllabus(2007), with reference to the current recommendations for the teaching of statistics. So that, it was performed the content analysis of the two syllabuses, firstlyat a structural level and, then, focused on the topic Organisation and Data Processing. Concerning the results, unlike the previous syllabus, the 2013 program omits methodological guidelines and resources, which translates into no reference to research projects, to teamwork and to calculators and computers, highlighting a regression regarding the recentre commendations to approach this topic.
\end{abstract}

Key words: Math syllabus; basic education, organization and data processing. 


\section{Introdução}

Reconhecer a importânciado desenvolvimento da competência de usar e interpretar dados para o exercício de uma cidadania crítica e para a promoção do raciocínio e sentido estatístico, seja na tomada de decisões pessoais, no trabalho e em outras áreas científicas (BURRIL;BIEHLER, 2011), está na origem da crescente importância que tem sido dada às questões do ensino e aprendizagem da estatística.

Em estatística o aluno explora diferentes tipos de raciocínio, quando elabora ou analisa um questionário, seleciona uma amostra e trata os dados recolhidos. Estas ações implicam a organização de dados, a escolha da forma mais apropriada para os representar,a sua interpretação com a ajuda de diferentes medidas estatísticas e a validação das conclusões que daí tira, atividades que desenvolvem nos alunos, nomeadamente no ensino básico, capacidades de recolha, organização e tratamento, representação e resumo de dados (SCHIELD, 2004). O autor acrescenta que, de forma mais específica, a organização e tratamento de dados tem como objetivo principal possibilitar aos alunos a construção e o desenvolvimento das capacidades necessárias para produzir, utilizare interpretar informação numérica, pois, através dos primeiros contactos com a produção e utilização de tabelas e de representações gráficas, o aluno implementa os primeiros instrumentos de estatística descritiva, em particular a noção de resumo estatístico, continuando com o estudo de algumas estatísticas de posição e de dispersão.

Ora, a produção, recolha e análise da informação estatística implica fazer escolhas que acentuam ou atenuam determinados aspetos em detrimento de outros. Espera-seque as escolhas feitas promovam o estudo desta temática,de modo a contribuir para o desenvolvimento do espírito crítico, indispensável para o cotidiano de qualquer cidadãoe paraprecaver usos enviesados e abusivos da estatística. Olh@res, Guarulhos, v. 3, n. 1, p. 280-305. Maio, 2015. 
Alterações do programa de matemática do ensino básico português: o caso do tema organização e tratamento de dados

Os vários materiais disponíveis para o ensino e aprendizagem da estatística (e não só)influenciamo ensino dos professores e a aprendizagem dos alunos, como é o caso do Programa de Matemática para o Ensino Básico ${ }^{1}$ (MINISTÉRIO DA EDUCAÇÃO E CIÊNCIA, 2013). Neste caso, o caráter mais acentuadamente prescritivo de algumas dimensões do programa escolar poderá ter um grande impacto sobre o ensino e a aprendizagem da estatística: diretamente sobre o ensino dos professores, os manuais escolares e os exames e, indiretamente, sobre a aprendizagem dos alunos (VERGNES, 2001).

Algumas investigações que incidem sobre o ensino e a aprendizagem da estatística (ROUAN, 2003) evidenciam que a elaboração de situações didáticas que mobilizem os saberes estatísticos e probabilísticos atenuam um conjunto considerável de dificuldades que os alunos revelam e os professores enfrentam, tais como, a construção, a leitura e interpretação de parâmetros, de representações gráficas e as modelizações.Essas dificuldades e obstáculos mostram a importância de realizar estudos curriculares para dar mais visibilidade ao lugar da estatística nos programas do ensino básico,justifica-se, assim, o estudo que apresentamos. Analisam e discutem-se as alterações verificadas no Programa de Matemática para o Ensino Básico (MINISTÉRIO DA EDUCAÇÃO E CIÊNCIA, 2013), em relação ao programa anteriormente em vigor (MINISTÉRIO DA EDUCAÇÃO, 2007), no que concerne ao tema Organização e Tratamento de Dados. Nessa análise, confrontamos as orientações curriculares dos programas com as recomendações atuais para o ensino e aprendizagem da estatística, que constam da literatura da especialidade.

${ }^{1}$ Em Portugal, o ensino básico desenvolve-se ao longo dos primeiros nove anos de escolaridade e estrutura-se em três ciclos: $1 .^{\circ}$ ciclo (do $1 .^{\circ}$ ano ao $4 .^{\circ}$ ano); $2 .^{\circ}$ ciclo $\left(5 .^{\circ}\right.$ e $6 .^{\circ}$ anos) e $3 .^{\circ}$ ciclo (do $7 .^{\circ}$ ano ao $9 .^{\circ}$ ano).

Olh@res, Guarulhos, v. 3, n. 1, p. 280-305. Maio, 2015. 


\section{Orientações atuais para o ensino e aprendizagem da estatística}

De entre a multiplicidade de domínios relevantes a ter em conta no ensino e aprendizagem da estatística, referir-nos-emos, de seguida, aos domínios do conteúdo, das tarefas, dos recursos e da avaliação.

\subsection{Conteúdo}

Burril e Biehler (2011), baseando-se em diferentes perspetivas sobre o ensino da estatística, designadamente a estrutura para o pensamento estatístico de Wild e Pfannkuch (1999), a visão da estatística como um processo diferente da matemática, a literacia estatística e a estocástica, e adaptando os critérios de Heymann e de Heitele para as ideias fundamentais em matemática e em estocástica, respetivamente, estabeleceram sete ideias estatísticas fundamentais:dados, entendidos como números num contexto: tipos eformas de recolha de dados;variação: identificar e medir a variabilidade dos dados para prever, explicar ou controlar;distribuição: noções de tendências e dispersão que fundamentam o raciocínio acerca de distribuições empíricasde variáveis estatísticas e de distribuições teóricas de variáveis aleatórias, e resumos de distribuições amostrais;representação: gráfica ou outrotipo de representação e transformações de representações (transnumeração), de modo a revelar diferentes aspetos dos dados; associação e relações de modelação entre duas variáveis: relações entre variáveis categoriais e dados numéricos, incluindo a regressão para modelar associações estatísticas; modelos de probabilidade para processos de geração de Olh@res, Guarulhos, v. 3, n. 1, p. 280-305. Maio, 2015. 
Alterações do programa de matemática do ensino básico português: o caso do tema organização e tratamento de dados

dados: modelar relações estruturais hipotéticas com origem numa teoria, simulações ou grandes quantidades de dados, quantificando a variabilidade dos dados e a sua estabilidade a longo termo; e amostragem e inferência:relação entre amostras e população e extração de conclusões dos dados com algum grau de incerteza.

Por outro lado, osmesmos autores preconizam que os professores e os alunos devem desenvolver hábitos estatísticos, tais como: usar dados reais, em que se salienta a variação e o ruído que levam a ter em atenção a origem dos dados; construir intuições, usando simulações para gerar distribuições, prever antes de calcular e formular questões sobre o acaso envolvendo os dados; começar com um gráfico para investigar associações e analisar diferentes representações de distribuições; explorar representações alternativas dos dadospara contrastar o que pode ser aprendido sobre a forma, a tendência central e a dispersão de distribuições e relações e conexões entre variáveis; investigar e explorar antes de introduzir fórmulas, recorrendo a simulações para modelar distribuições de probabilidade, jogar com o acaso e experienciar a variabilidade; e usar projetos e experimentos para envolver os alunos em "fazer" estatística.

Os conteúdos, antes referidos, enfatizam a estatística como domínio do saber escolar que se aproxima do trabalho do próprio estatístico e com potencialidades para a promoção de uma atitude crítica por parte dos aprendizes. Simultaneamente, os conteúdos enumerados não limitam a estatística a uma visão apenas tecnicista e teórica, frequentemente veiculada na escola, e que explica o facto de professores e alunos a verem como tema mais fácil de ensinar e aprender comparativamente com outros temas matemáticos (FERNANDES; SOUSA; RIBEIRO, 2004,FERNANDES; CARVALHO; CORREIA, 2011). 


\subsection{Tarefas}

Decorre do que foi referido antes, que a comunidade de educação estatística vem valorizando, na aprendizagem da estatística, o recurso a tarefas envolvendo dados reais, relativos aos próprios alunos ou com eles relacionados (FERNANDES; CARVALHO; RIBEIRO, 2007,MACGILLIVRAY; PEREIRA-MENDONZA, 2011). O recurso a esse tipo de tarefa, em que o contexto é particularmente saliente,reveste-se da maior importância em termos do desenvolvimento de significado e da própria estatística. Nesse último caso, a perspetiva da estatística comouma ciência dos números em contexto (MOORE, 1992)é da maior relevância para o envolvimento dos alunos na aprendizagem, motivando-os. Além disso, o conhecimento do contexto pode desempenhar um papel clarificador em muitas fases de um estudo estatístico, sobretudo no que concerne à fase de interpretação dos resultados.

De entre os diferentes tipos de tarefas, os projetos de natureza investigativa assumem-se como experiências de aprendizagem com um potencial específico,ao constituírem "veículos ideais para o envolvimento do aluno na aprendizagem de resolução de problemas em contexto e para sintetizar componentes da aprendizagem" (MACGILLIVRAY; PEREIRA-MENDONZA, 2011, p. 109), o quenão é imediatamente inerente a qualquer outro tipo de tarefa. Também Bataneroet al. (2011) justificam o recurso a projetos no ensino da Estatística, pelo facto deste tipo de tarefa aumentar a motivação dos alunos e relevar o contexto e a natureza realista das tarefas.

Olh@res, Guarulhos, v. 3, n. 1, p. 280-305. Maio, 2015. 
Alterações do programa de matemática do ensino básico português: o caso do tema organização e tratamento de dados

Este tipo de tarefa constitui um contexto natural para os alunos experienciarem o processo de realização de inquirições estatísticas reais, através da escolha de uma problemática, estabelecimento de um plano, recolha e exploração de dados e formulaçãode conclusões. Estas fases de exploração dos projetos investigativos, embora possam divergir ligeiramente de autor para autor, partilham uma estrutura subjacente ao ciclo investigativo de Wild e Pfannkuch (1999): Problema; Plano; Dados; Análise e Conclusão (PPDAC).

Segundo MacGillivray e Pereira-Mendonza (2011), os projetos investigativos devem também fazer parte da formação dos professores que ensinam estatística, avançando, para tal, quatro recomendações: 1) os projetos estatísticos devem fazer parte da educação matemática dos programas de formação dos futuros e atuais professores; 2) os projetos estatísticos devem enfatizar o ciclo investigativo como meio de ensinar o pensamento estatístico e desenvolver a compreensão e conhecimentos estatísticos; 3 ) os projetos a realizar pelos futuros e atuais professores devem, sempre que possível, poder ser adaptados ao uso escolar; e 4) os professores necessitam de realizar as mesmas experiências de aprendizagem que, mais tarde, vão propor aos seus alunos.

O trabalho em grupo dos alunos está estreitamente relacionado com os projetos investigativos pois "um forte sentido de pertença ao grupo facilita o trabalho em equipa à medida que o projeto se desenvolve através da obtenção de dados, exploração e análise (se apropriado), interpretação e relato no contexto" (MACGILLIVRAY;PEREIRAMENDONZA, 2011, p. 113). Além disso, a aprendizagem colaborativa, que lhe está associada, fornece oportunidades aos alunos de diferentes capacidades e níveis escolares, podendo ainda facilitar 
aos professores a obtenção de informação quando observam e apoiam o trabalho dos alunos.

Com base em vários estudos, Petocz e Reid (2007) identificaram vários aspetos positivos do trabalho de grupo, especificamente:permitir aos professores desenvolver tarefas mais compreensivas;partilhar e discutirdiferentes pontos de vista dos membros do grupo; possibilitar aos alunos praticar dinâmicas e processos de grupo; desenvolver competências interpessoais; e promover a reflexão e a discussão, como parte essencial do processo de se tornarem práticos competentes e reflexivos.

No caso do estudo de Fernandes, Júnior e Vasconcelos (2013), em que os alunos desenvolveram projetos de tipo investigativo, trabalhando em pares ou pequenos grupos, "todos os alunos afirmaram que o trabalho com os seus colegas foi importante para ultrapassar dúvidas e dificuldades" e "referiram-se à ajuda recebida e/ou indicaram situações em que essa ajuda permitiu avançar na resolução das tarefas" (p.106).

\subsection{Recursos}

De entre os diferentes recursos passíveis de serem usados no ensino da estatística, iremos referir-nos particularmente às novas tecnologias, incluindo calculadoras, computadores e a internet, cujo uso é mesmo mais enfatizado no caso da estatística do que em outras áreas da matemática.

Para Jolliffe (2007), a chamada revolução tecnológica está na origem das maiores alterações no ensino da estatística, ao permitir aos alunos resolver problemas reais com dados reais erelatar os resultados Olh@res, Guarulhos, v. 3, n. 1, p. 280-305. Maio, 2015. 
Alterações do programa de matemática do ensino básico português: o caso do tema organização e tratamento de dados

obtidos de uma maneira que não era realizável no passado.Por outro lado, os educadores acreditam que o uso de dados reais em tópicos de interesse dos alunos contribuem para as suas motivações em aprenderem estatística e para gostarem de o fazer(FERNANDES; CARVALHO; CORREIA, 2011).

Segundo Ben-Zvi (2000), são vários os atributos dos computadores que parecem contribuir parao desenvolvimento dos sentido e significados dos alunos, nomeadamente: a capacidade deoperar de forma rápida e precisa; ligar dinamicamente múltiplas representações; simplificar procedimentos; fornecer feedback e transformar uma representação como um todo numobjeto manipulável. Para este autor, as representações como um todo, podendo ser editadas,transformadas, combinadas, separadas em partes, armazenadas, evocadas etc., "implicamuma reorganização da atividade cognitiva e uma mudança do foco de atenção para um nívelcognitivo superior” (p. 141).

Em Fernandes, Júnior e Vasconcelos (2013) refere-se que, "na realização das tarefas, o uso da tecnologia, e especialmente da folha de cálculo, foi a componente da estratégia mais positivamente valorizada pelos alunos", acrescentando-se que, "unanimemente, os alunos consideraram a folha de cálculo uma ferramenta útil na aprendizagem da estatística, destacando agora a facilidade da construção de tabelas e gráficos, o controlo de erros, a rapidez e os cálculos"(p. 108).

Também Pratt, Davies e Connor (2011) discutem potencialidades do uso da tecnologia no ensino da estatística, referindo: usar representações como instrumentos dinâmicos de análise; expressar modelos pessoais; explorar modelos; armazenar e processar dados reais e partilhar e comunicar.

Olh@res, Guarulhos, v. 3, n. 1, p. 280-305. Maio, 2015. 
Estes atributos da tecnologia alteram as assunções acerca do que deve ser aprendido e implicam queo currículo de estatística seja reformulado de acordo, incluindo os materiais de ensino, aspráticas de sala de aula e a forma de os alunos aprenderem. Neste sentido, Moore (1997)perspetiva as ferramentas tecnológicas como meios de apoiar os alunos na construção ativado conhecimento, proporcionar oportunidades para os alunos refletirem sobre fenómenosobservados, ajudar os alunos a desenvolver capacidades metacognitivas e renovar o ensino eo currículo na base de fortes sinergias entre conteúdo, pedagogia e tecnologia.

\subsection{Avaliação}

Em Garfield e Franklin (2011) refere-se que a avaliação repousa em três pilares: 1) modelo de como os alunos representam o conhecimento e desenvolvem competências no domínio do conteúdo (cognição); 2) tarefas ou situações que permitam observar a performance do aluno (observação); e 3) um método de interpretação para fazer inferências a partir da performance observada (interpretação). Ligados a estes pilares, examinam-se os propósitos e usos da avaliação do aluno nas três categorias seguintes: avaliação $d a$ aprendizagem, relacionada com a avaliação sumativa; avaliação para a aprendizagem, relacionada com a avaliação formativa; e avaliação como aprendizagem, englobando métodos sumativos e formativos e que coloca o aluno entre a aprendizagem e a avaliação.

3. 
Alterações do programa de matemática do ensino básico português: o caso do tema organização e tratamento de dados

No presente estudoanalisam-se as principais alterações verificadas no Programa de Matemática para o Ensino Básico em vigor (MINISTÉRIO DA EDUCAÇÃO E CIÊNCIA, 2013) em relação ao programa anterior (MINISTÉRIO DA EDUCAÇÃO, 2007),com especial incidência no tema de Organização e Tratamento de Dados.

Tendo em conta a própria organização dos dois programas objeto de estudo, estabeleceram-se dois níveis para a sua análise: as alterações estruturais do programa; e as alterações no tema Organização e Tratamento de Dados.As alterações estruturais referem-se à globalidade do ensino básico, dizendo respeito aos vários temas matemáticos e, portanto, também ao tema de Organização e Tratamento de Dados, tendo essa análise sido orientada pelas dimensões: finalidades; objetivos gerais de aprendizagem; temas matemáticos; capacidades transversais; indicações metodológicas; recursos eavaliação das aprendizagens.

As alterações no tema Organização e Tratamento de Dados focam-se neste tema matemático e essa análise foi orientada pelas dimensões: gestão do tempo; objetivos gerais; conteúdos; indicações metodológicas e recursos.Aavaliaçãoe interpretação das alterações verificadas no programa de 2013 , relativamente ao programa de 2007 , sobretudo no que se refere ao tema Organização e Tratamento de Dados, serão orientadas, também, pelas recomendações atuais para o ensino e aprendizagem da estatística que são preconizadas na literatura.

4. Alterações do programa de matemática para o ensino básico no tema Organização e Tratamento de Dados

Olh@res, Guarulhos, v. 3, n. 1, p. 280-305. Maio, 2015. 
Em termos dos resultados da análise realizada aos programas escolares, começamos por descrever as suas alterações estruturais, seguindo-se a apresentação das alterações específicas no tema Organização e Tratamento de Dados.

\subsection{Alterações estruturais do programa}

Emnível estrutural, o Programa de Matemática para o Ensino Básico (MINISTÉRIO DA EDUCAÇÃO E CIÊNCIA, 2013) apresenta alterações ao nível das finalidades, dos objetivos gerais de aprendizagem, dos temas matemáticos; das capacidades transversais, das indicações metodológicas e dos recursos.

No caso das finalidades, as duas finalidades do programa de 2007 - 1) Promover a aquisição de informação, conhecimento e experiência em Matemática e o desenvolvimento da capacidade da sua integração e mobilização em contextos diversificados e 2) Desenvolver atitudes positivas face à Matemática e a capacidade de apreciar esta ciência foram substituídas pelas três seguintes finalidades no programa de 2013: 1) A estruturação do pensamento; 2) A análise do mundo natural; e 3) A interpretação da sociedade. Assim, em termos de diferenças, salientam-se os aspetos lógicos da matemática na finalidade 1) do atual programa e não se explicitam os aspetos afetivos que constavam da finalidade 2) do programa anterior.

Em ambos os programas são estabelecidos os objetivos gerais de aprendizagem em cada um dos ciclos de ensino, salientando-se o Olh@res, Guarulhos, v. 3, n. 1, p. 280-305. Maio, 2015. 
Alterações do programa de matemática do ensino básico português: o caso do tema organização e tratamento de dados

carácter genérico e a abrangência crescente desses objetivos com os ciclos de ensino no programa de 2013:1) identificar/designar (objetos matemáticos); 2) estender (generalização); 3) reconhecer (validação); 4) saber (conhecimento); 5) reconhecer, dado... (justificar com casos); 6) provar/demonstrar (demonstração); 7) justificar (evocação).De entre estes objetivos, os quatro primeiros são comuns aos três ciclos de ensino e os três últimos são relativos apenas ao $3 .^{\circ}$ ciclo. Diferentemente, no programa de 2007 os objetivos são formulados para cada um dos temas matemáticos de cada ciclo, portanto mais centrados nesses temas, enquanto no programa de 2013 são de natureza transversal e sem referência aos temas matemáticos. Além disso, no programa de 2007 também é indicado o propósito principal de ensinode cada tema matemático,em cada nível de ensino.

Entre os temas matemáticos(Números e Operações, Geometria e Medida, Álgebra e Organização e Tratamento de Dados), agora designados domínios de conteúdo,não se salientam grandes diferenças entre os dois programas. O tema Álgebra não é considerado de forma explícita no $1 .^{\circ}$ ciclo em ambos os programas e no programa de 2013 é estabelecido o novo tema matemático Funções, Sequências e Sucessões (FSS),no 3. ${ }^{\circ}$ ciclo, e que no programa anterior estava incluído no tema Álgebra. A este nível, a maior diferença reside no desenvolvimento dos temas matemáticos, que no programa de 2007 se realiza, fundamentalmente, por ciclo de ensino básico (os quatro anos de escolaridade do $1 .^{\circ}$ ciclo são agrupados em dois subníveis:1. ${ }^{\circ}$ e $2 .^{\circ}$ anos; 3. ${ }^{\circ}$ e $4 .^{\circ}$ anos), enquanto no programa de 2013 esse desenvolvimento se concretiza por ano de escolaridade. Também em termos da informação disponibilizada nos temas se observam diferenças: no programa de 2007, para cada tema matemático, são especificados tópicos, objetivos específicos e notas clarificadoras do conteúdo e da metodologia; já no programa de 2013 apenas são referidos os conteúdos relativos a cada um dos tópicos considerados e Olh@res, Guarulhos, v. 3, n. 1, p. 280-305. Maio, 2015. 
é mencionado, a título indicativo, o número de tempos (de 45 minutos) que deve ser dedicado a cada tema matemático.Assim, a gestão do programa de 2007 permite uma maior autonomia à escola e ao professor, ao mesmo tempo que é mais informativo do que o programa de 2013, onde se destacam mais os conteúdos e se assume uma natureza mais prescritiva destas orientações curriculares.

Às capacidades transversais estabelecidas no programa de 2007 (resolução de problemas, raciocínio matemático e comunicação) são acrescentadas no programa de 2013 as duas seguintes: "conhecimento de factos e de procedimentos" e "a matemática como um todo coerente", perspetivadas como aquisições integradas a partir dos objetivos gerais de aprendizagem.Nessas novas capacidades salientase o papel da memória e da lógica, essa última já destacada ao nível das finalidades.

No caso das indicações metodológicas, no programa de 2007 são apresentadas muitas sugestões em cada tema matemático de cada nível de escolaridade, organizadas segundo a abordagem, as tarefas e os conceitos específicos. Já no programa de 2013 não são apresentadas quaisquer sugestões metodológicas, inferindo-me mesmo uma visão negativa sobre a explicitação de tais sugestões, conforme se constata no seguinte excerto do programa (MINISTÉRIO DA EDUCAÇÃO E CIÊNCIA, 2013).

A experiência acumulada dos professores e das escolas
é um elemento fundamental no sucesso de qualquer
projeto educativo, não se pretendendo, por isso,
espartilhar e diminuir a sua liberdade pedagógica nem
condicionar a sua prática letiva. Pelo contrário, o
presente Programa reconhece e valoriza a autonomia
dos professores e das escolas, não impondo
metodologias $\quad$ específicas.

Olh@res, Guarulhos, v. 3, n. 1, p. 280-305. Maio, 2015. 
Alterações do programa de matemática do ensino básico português: o caso do tema organização e tratamento de dados

Osrecursossão referidos a diferentes níveis do programa de 2007, salientando-se a referência sistemática que lhes é dada no desenvolvimento de cada tema matemático dos diferentes ciclos escolares. Especificamente, referem-se os materiais manipuláveis, os instrumentos de desenho (régua, esquadro e compasso), calculadoras e computadores. No caso das calculadoras e computadores recomendase o seu uso ao longo de todos os ciclos, afirmando-se que

O seu uso é particularmente importante na resolução de problemas e na exploração de situações, casos em que os cálculos e os procedimentos de rotina não constituem objetivo prioritário de aprendizagem, e a atenção se deve centrar nas condições da situação, nas estratégias de resolução e na interpretação e avaliação dos resultados. (MINISTÉRIO DA EDUCAÇÃO, 2007, pp. 9-10)

No programa de 2013, tal como no caso das metodologias, deixa-se a seleção dos recursos ao critério da escola e dos professores. Além das calculadoras, em que é recomendado oseu uso mais restritivo e em níveis escolares mais avançados, não é feita qualquer referência a outros recursos. Nesse sentido, afirma-se que

o uso da calculadora no Ensino Básico apenas é expressamente recomendado em anos escolares mais avançados e sobretudo em situações pontuais de resolução de problemas que envolvam, por exemplo, um elevado número de cálculos, a utilização de valores aproximados, operações de radiciação ou a determinação de razões trigonométricas ou de amplitudes de ângulos dada uma razão trigonométrica, quando não haja intenção manifesta de, por alguma razão justificada, dispensar esse uso. (MINISTÉRIO DA EDUCAÇÃO E CIÊNCIA, 2013, pp. 29-30)

Olh@res, Guarulhos, v. 3, n. 1, p. 280-305. Maio, 2015. 
Finalmente, no programa de 2007 preconiza-se uma avaliação das aprendizagens pautada pelos princípios de congruência com o programa, constituindo uma parte integrante do processo de ensino e aprendizagem, assentando na diversidade de formas e instrumentos de avaliação, assumindo um propósito predominantemente formativo, decorrendo num clima de confiança e sendo transparente para os alunos e para as suas famílias. No programa de 2013 remete-se a questão da avaliação para os normativos legais em vigor, destacandose as metas curriculares como referencial de avaliação e as funções da avaliação enquanto regulação e orientação do percurso de aprendizagem.

\subsection{Alterações no tema Organização e Tratamento de Dados}

No âmbito do tema Organização e Tratamento de Dados destacam-se alterações emnível da gestão do tempo, dos objetivos gerais, dos conteúdos, das indicações metodológicas e dos recursos.

No programa de 2013 é sugerida, a título de indicação, a distribuição dos tempos escolares pelos vários temas matemáticos em cada ano escolar do $2 .^{\circ}$ e $3 .^{\circ}$ ciclos, o que não acontece no programa de 2007. Embora sem caráter prescritivo, os tempos sugeridos no programa para cada tema matemático tendem a influenciar a importância dada ao respetivo tema, seja pelos professores, pelos manuais escolares ou mesmo pelos alunos. 
Alterações do programa de matemática do ensino básico português: o caso do tema organização e tratamento de dados

Considerando o total dos tempos propostos conjuntamente no $2 .^{\circ}$ e $3 .^{\circ}$ ciclos, apresentam-se,na Tabela 1, as percentagens dos tempos (de 45 minutos) de cada tema matemático do programa,relativas ao $2 .^{\circ}$ ciclo, ao $3 .^{\circ}$ ciclo e ao total dos dois ciclos.

Tabela 1 - Tempos totais sugeridos (em \%) nos temas matemáticos no2. ${ }^{\circ}$ ciclo, $3 .^{\circ}$ ciclo e total

$\%$ de tempos totais

Temas matemáticos

2. ${ }^{\circ}$ ciclo $\quad 3 .^{\circ}$ ciclo Total

Números e Operações (NO)

12

7

19

Geometria e Medida (GM)

18

21

39

Álgebra (ALG)

9

25

34

Organização e Tratamento de Dados (OTD)

4

4

8

Nota: No caso do $3 .^{\circ}$ ciclo incluíram-se os tempos relativos ao tema Funções, Sequências e Sucessões (FSS) no tema Álgebra (ALG).

Pela Tabela 1 constata-se que as percentagens de tempos sugeridaspara o tema de OTD são iguais no $2 .^{\circ}$ e $3 .^{\circ}$ ciclos $(4 \%)$ e muito inferiores às dos outros temas matemáticos.No total, menos de metade do que no tema NO, que é o tema matemático imediatamente a seguir.

Olh@res, Guarulhos, v. 3, n. 1, p. 280-305. Maio, 2015. 
A questão do tempo sugerido em OTD agrava-se ainda mais, na medida em que nesse tema se incluem conteúdos de "Gráficos cartesianos" no $5 .^{\circ}$ ano e, embora sem interferir nas percentagens da Tabela 1, de "Representação de conjuntos" no $1 .^{\circ}$ e $2 .^{\circ}$ anos.

Tal como foi referido antes para a estrutura do programa, também no tema OTD são referidos objetivos gerais em ambos os programas, centradosnaestatística no programa de 2007 e genéricos, abstratos e comuns aos vários temas matemáticos, portanto sem referência à estatística, no caso do programa de 2013.

Emnível dos conteúdos, não se destacam diferenças relevantes entre os dois programas, abordando-se essencialmente os mesmos conteúdos. No caso do programa de 2013, os conteúdos de Probabilidades estão concentrados no $9 .^{\circ}$ ano e3..$^{\circ}$ ciclo de ensino básico, enquanto no programa de 2007 esses conteúdos são desenvolvidos ao longo dos três ciclos do ensino básico.Ainda no domínio das Probabilidades, no programa de 2013 refere-se a "Comparação de probabilidades com frequências relativas em experiências aleatórias em que se presume a equiprobabilidade dos casos possíveis" (MINISTÉRIO DA EDUCAÇÃO E CIÊNCIA, 2013, p. 27). Ora, autilização do conceito frequencista de probabilidade apenas nessas situações reduza sua aplicabilidade aoscasos em que é possível usar o conceito clássico de probabilidade. Embora podendo estar implícita a possibilidade de controlar as estimativas empíricas das probabilidadesatravés das probabilidades teóricas, que podem ser estabelecidasa priori, tal contraria a natureza do próprio conceito frequencista de probabilidade, especificamente ao não clarificar que esta definição de probabilidade pode aplicar-se a experiências em que os acontecimentos são ou não equiprováveis (FERNANDES,1999).

Já emnível das indicações metodológicas a situação é diversa, pois, no programa de 2013, tal como foi referido antes para a estrutura do Olh@res, Guarulhos, v. 3, n. 1, p. 280-305. Maio, 2015. 
Alterações do programa de matemática do ensino básico português: o caso do tema organização e tratamento de dados

programa, assume-se,explicitamente, a não especificação de sugestões metodológicas como forma de promoção da autonomia das escolas e dos professorese, em última instância, a melhoria da aprendizagem dos alunos.

Diferentemente, no programa de 2007, no tema matemático OTD, tal como nos restantes temas matemáticos, são feitas muitas sugestões metodológicas, seja em termos de abordagem, seja em termos de tarefas, como se salienta na Tabela 2.

Tabela 2 - Indicações metodológicas no tema OTD ao nível da abordagem e das tarefas no programa de 2007, segundo os níveis de escolaridade

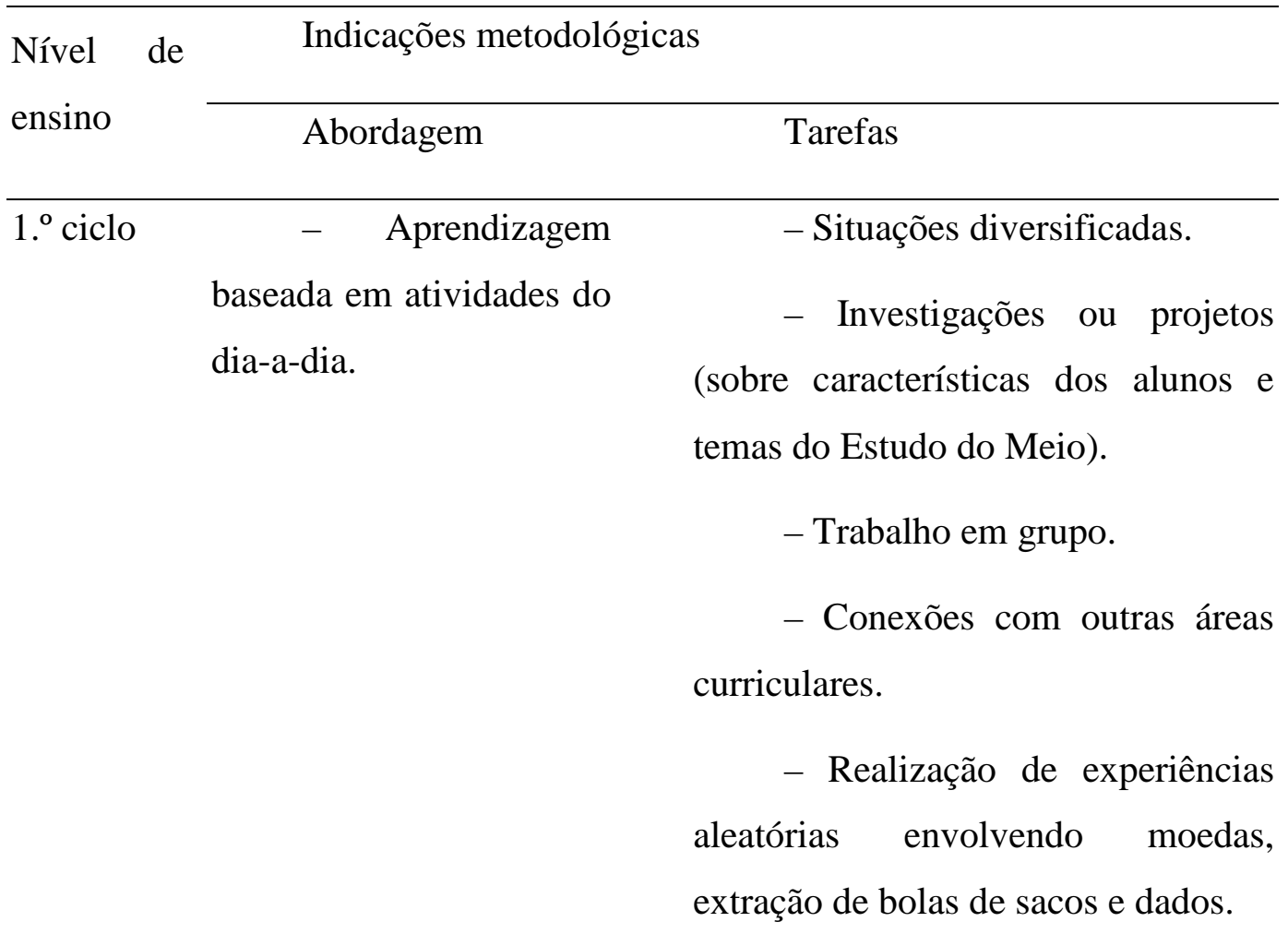

Olh@res, Guarulhos, v. 3, n. 1, p. 280-305. Maio, 2015. 


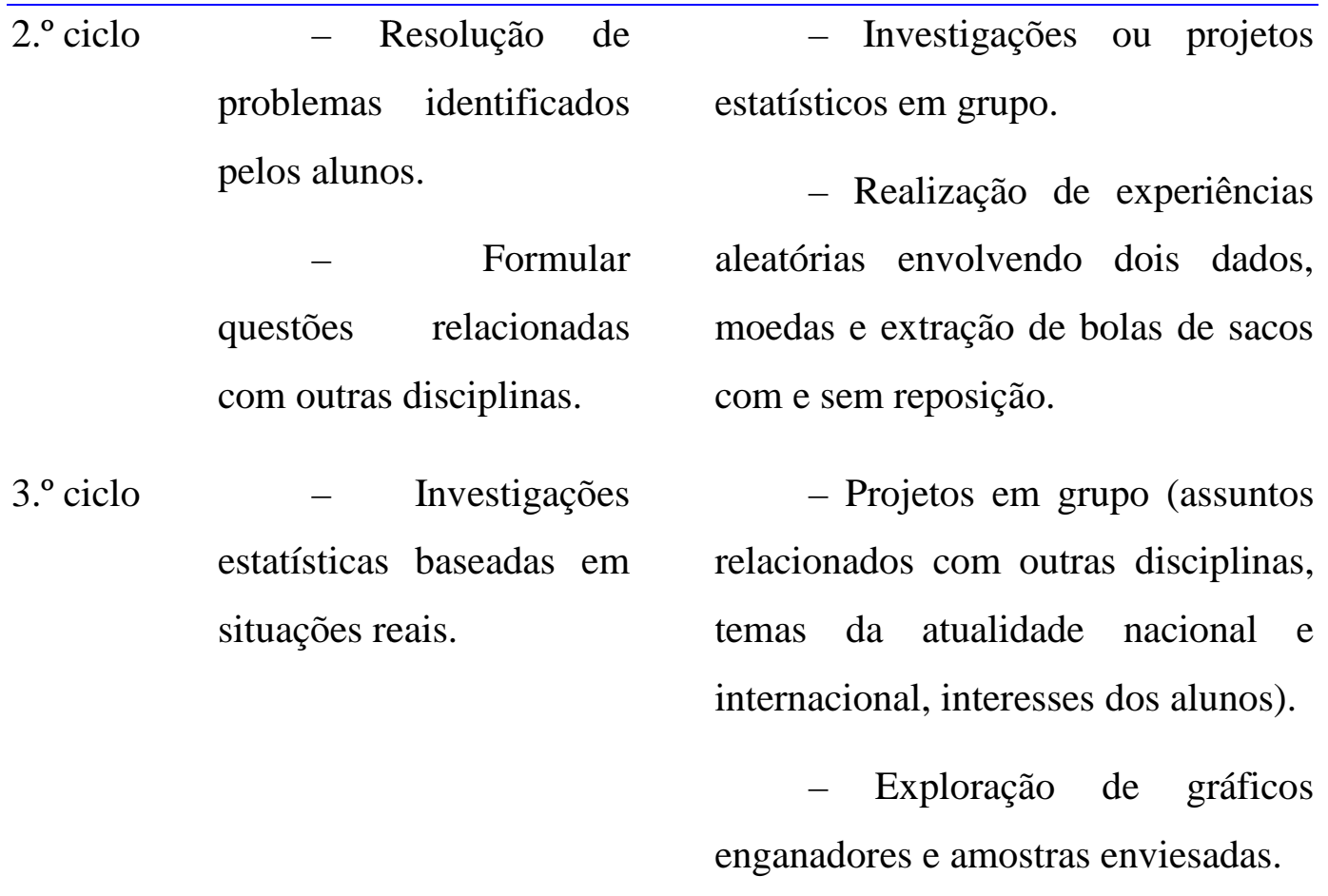

Da Tabela 2 destaca-se a recomendação da realização de investigações ou projetos estatísticos em todos os três níveis de escolaridades, desenvolvidos pelos alunos em pequenos grupos.O destaque dado a este tipo de tarefas decorre ainda dos tópicos elencados neste tema matemático no $3 .^{\circ}$ ciclo: planeamento estatístico e tratamento de dados.

Por outro lado, preconiza-se a aprendizagem da estatística com base em atividades relacionadas com o dia-a-dia, com outras disciplinas e com os interesses dos próprios alunos, o que enfatiza o significado e a utilidade da estatística. Simultaneamente, a realização de atividades nestes contextos pode contribuir para o desenvolvimento deum mais profundo sentido crítico e de cidadania do aluno. Em relação ao sentido crítico, ele é referido explicitamente no programa, através da exploração de gráficos enganadores e amostras enviesadas. 
Alterações do programa de matemática do ensino básico português: o caso do tema organização e tratamento de dados

Em termos de recursos, salienta-se a referência ao uso de calculadoras e computadores no $2 .^{\circ}$ e $3 .^{\circ}$ ciclos, aludindo à folha de cálculo e à internet. Além destes recursos tecnológicos, no caso das Probabilidades, é também recomendado o uso de objetos aleatórios como moedas, dados e sacos de bolas, bem como tabelas e diagramas de árvore, os quais se revelam particularmente adequados para descrever o espaço amostral e determinar o número de casos favoráveis e possíveis, tendo em vista a determinação da probabilidade na perspetiva clássica.O recurso a tabelas e diagramas de árvore para determinar e comparar probabilidades de acontecimentos compostos também é referido no programa de 2013.

\section{Conclusão}

Nas finalidades e capacidades transversais do programa de 2013, relativamente ao programa anterior,infere-se uma maior ênfase do papel da memorização na aprendizagem e uma perspetiva mais formal da matemática. Por outro lado, a não referência à finalidade afetiva, que constava do programa anterior, parece não reconhecer o seu contributo para a aprendizagem.Ora,o nível de destaque dado a estes aspetos no programa mostra a importância que lhe é atribuída.

A não inclusão de recomendações metodológicas no programa de 2013 pode ter por consequência a desvalorização das orientações atuais para o ensino da matemática. No caso da estatística, destaca-se a ausência de referência aos projetos investigativos e a tarefas que envolvam dados reais e relativos aos alunos ou relacionados com os seus interesses, que são unanimemente recomendados pelos investigadores na área da educação estatística (e.g., 
BATANERO;ETAL., 2011,FERNANDES; CARVALHO; RIBEIRO, 2007,MACGILLIVRAY; PEREIRA-MENDONZA, 2011).

Por outro lado, à realização de projetos investigativos está geralmente associado o trabalho de grupo dos alunos, forma de trabalho que também não está prevista no programa de 2013 e que évista pelos professores como particularmente adequada no caso da estatística (FERNANDES;CARVALHO; CORREIA, 2011).

Tal como no caso das recomendações metodológicas, também no programa de 2013 são quase inexistentes as referências aos recursos a usar no ensino, advogando-se um uso mais restritivo da calculadorae não fazendo qualquer alusão ao computador. Ora, o uso destas tecnologias é amplamente preconizado no ensino da estatística (e.g., BEN-ZVI, 2000,PRATT;DAVIES; CONNOR, 2011).

Em síntese, no programa de 2013 omitem-se importantes recomendações atuais para o ensino e aprendizagem da estatística que constavam do programa anterior, representando um retrocesso em relação ao que é preconizado pelas várias instâncias de educação estatística. Simultaneamente ignorar a muita investigação que ultimamente tem sido desenvolvida no campo da didática da estatísticaparece contribuir para a desvalorizaçãodesse domínio de conhecimento, podendo também levar à não aplicação dos seus ensinamentos para uma melhor aprendizagem dos alunos.

Considerando, ainda, o desenvolvimento recente do ensino da estatística,em nível internacional, teremos de concluir pela importância de uma reestruturação substancial, quer relativa aos domínios do conteúdo, das tarefas e dos recursos, quer às abordagens pedagógicas a preconizar para este nível de ensino, que deveriam promover um grande envolvimento dos alunos. 
Alterações do programa de matemática do ensino básico português: o caso do tema organização e tratamento de dados

\section{Agradecimento}

Este trabalho contou com o apoio de Fundos Nacionais através da FCT - Fundação para a Ciência e a Tecnologia no âmbito do projecto PEst-OE/CED/UI1661/2014 do CIEd-UM e do proyecto Significados de la probabilidad en el currículo de la enseñanza obligatoria y la formación de profesores con la referencia EDU201341141-P.

Olh@res, Guarulhos, v. 3, n. 1, p. 280-305. Maio, 2015. 
Referências:

BATANERO, C.; ET AL.Enseñanza de la Estadística a través de proyectos. In: BATANERO, C.; DÍAZ, C. (Eds.). Estadística con Proyectos. Granada: Universidad de Granada, 2011, p. 9-46.

BEN-ZVI, D. (2000). Toward understanding the role of technological tools in statistical learning. Mathematical Thinking and Learning, 2(1-2), p. 127-155, 2000.

BURRIL, G.; BIEHLER, R. Fundamental statistical ideas in the school curriculum and in training teachers. In: BATANERO,C.; BURRIL,G.; READING, C. (Eds.). Teaching statistics in school mathematics-Challenges for teaching and teacher education: $A$ joint ICMI/IASE study. New York: Springer, 2011, p. 57-69.

FERNANDES, J. A. Intuições e aprendizagem de probabilidades: uma proposta de ensino de probabilidades no $9 .^{\circ}$ ano de escolaridade. Tese de doutoramento. Braga: Universidade do Minho, 1999.

FERNANDES, J. A.; CARVALHO, C. F.; CORREIA, P. F. Contributos para a Caracterização do Ensino da Estatística nas Escolas. Boletim de Educação Matemática (BOLEMA), 24(39), p. 585-606, 2011.

FERNANDES, J. A.; CARVALHO, C.; RIBEIRO, S. A. Caracterização e implementação de tarefas de Estatística: um exemplo no 7. ano de escolaridade. Zetetiké, 15(28), p. 27-61, 2007.

FERNANDES, J. A.; JÚNIOR, A. P. O.; VASCONCELOS, A. P. Caracterização, implementação e avaliação de uma estratégia de ensino de estatística no $7 .^{\circ}$ ano. Perspectivas da Educação Matemática, v. 6, n. 11, p. 93-109, 2013.

FERNANDES, J. A.; SOUSA, M. V.; RIBEIRO, S. A. O ensino de estatística no ensino básico e secundário: Um estudo exploratório. In: FERNANDES,J. A.; SOUSA,M. V.; RIBEIRO, S. A. (Orgs.). Ensino e aprendizagem de probabilidades e estatística Actas do I Encontro de Probabilidades e Estatística na Escola. Braga: Centro de Investigação em Educação da Universidade do Minho, 2004, p. 165-193.

GARFIELD, J.; FRANKLIN, C. Assessment of learning, for learning, and as learning in statistics education.In: BATANERO,C.; BURRIL,G.; READING, C. (Eds.). Teaching statistics in school mathematics -Challenges for teaching and teacher education: A joint ICMI/IASE study. New York: Springer, 2011, p. 133-145.

JOLLIFFE, F. The changing brave new world of statistics assessment. In: PHILLIPS,B.; WELDON, L. (Eds.). The Proceedings of the ISI/IASE Satellite on Assessing Student Learning in Statistics.Voorburg: International Statistical Institute, 2007.

MACGILLIVRAY; PEREIRA-MENDONZA. Teaching statistical thinking through investigative projects. In: BATANERO,C.; BURRIL,G.; READING, C. (Eds.). Teaching statistics in school mathematics -Challenges for teaching and teacher education: A joint ICMI/IASE study. New York: Springer, 2011, p. 109-120. 
Alterações do programa de matemática do ensino básico português: o caso do tema organização e tratamento de dados

MINISTÉRIO DA EDUCAÇÃO E CIÊNCIA. Programa de matemática para o ensino básico. Lisboa: Autor, 2013.

MINISTÉRIO DA EDUCAÇÃO. Programa de matemática do ensino básico. Lisboa: Autor, 2007.

MOORE, D. S. New pedagogy and new content: The case of statistics. International Statistical Review, 65,p. 123-165, 1997.

MOORE, D. S. Teaching statistics as a respectable subject. In: GORDON,F.; GORDON, S. (Eds.). Statistics for the twenty-first Century.Washington, DC: The Mathematical Association of America, p. 14-25, 1992.

PETOCZ, P.; REID, A. Learning and assessment in statistics In: PHILLIPS,B.; WELDON, L. (Eds.). The Proceedings of the ISI/IASE Satellite on Assessing Student Learning in Statistics. Voorburg: International Statistical Institute, 2007.

PRATT, D.; DAVIES, N.; CONNOR, D.The role of technology in teaching and learning statistics. In: BATANERO,C.; BURRIL,G.; READING, C. (Eds.). Teaching statistics in school mathematics - Challenges for teaching and teacher education: $\mathrm{A}$ joint ICMI/IASE study. New York: Springer, 2011, p. 97-107.

ROUAN, O. Un graphique vaut-il mille nombres?Montréal: EBD, 2003.

SCHIELD, M. Information literacy, Statistical Literacy and Data Literacy.IASSISTQuarterly, 28 (2/3), p. 6-11, 2004.

VERGNES, D. Effets d'un stage de formation en géométrie. Recherches en Didactique des Mathématiques, 21(2), p. 99-121, 2001.

WILD, C.; PFANNKUCH, M. Statistical thinking in empirical enquiry (with discussion).International Statistical Review, 67(3), p. 223-265, 1999.

Recebido para publicação em 27 de outubro de 2014

Aprovado em 25 de fevereiro de 2015

Olh@res, Guarulhos, v. 3, n. 1, p. 280-305. Maio, 2015. 